\title{
Front Matter: Volume 11010
}

, "Front Matter: Volume 11010," Proc. SPIE 11010, Chemical, Biological, Radiological, Nuclear, and Explosives (CBRNE) Sensing XX, 1101001 (25 July 2019); doi: 10.1117/12.2537986

Event: SPIE Defense + Commercial Sensing, 2019, Baltimore, MD, United SPIE. States 


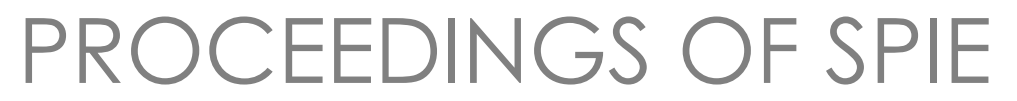

\section{Chemical, Biological, Radiological, Nuclear, and Explosives (CBRNE) Sensing $X X$}

Jason A. Guicheteau

Chris R. Howle

Editors

15-17 April 2019

Baltimore, Maryland, United States

Sponsored and Published by

SPIE

Volume 11010 
The papers in this volume were part of the technical conference cited on the cover and title page. Papers were selected and subject to review by the editors and conference program committee. Some conference presentations may not be available for publication. Additional papers and presentation recordings may be available online in the SPIE Digital Library at SPIEDigitalLibrary.org.

The papers reflect the work and thoughts of the authors and are published herein as submitted. The publisher is not responsible for the validity of the information or for any outcomes resulting from reliance thereon.

Please use the following format to cite material from these proceedings:

Author(s), "Title of Paper," in Chemical, Biological, Radiological, Nuclear, and Explosives (CBRNE) Sensing XX, edited by Jason A. Guicheteau, Chris R. Howle, Proceedings of SPIE Vol. 11010 (SPIE, Bellingham, WA, 2019) Seven-digit Article CID Number.

ISSN: 0277-786X

ISSN: 1996-756X (electronic)

ISBN: 9781510626850

ISBN: 9781510626867 (electronic)

Published by

SPIE

P.O. Box 10, Bellingham, Washington 98227-0010 USA

Telephone +1 3606763290 (Pacific Time) · Fax +1 3606471445

SPIE.org

Copyright (C) 2019, Society of Photo-Optical Instrumentation Engineers.

Copying of material in this book for internal or personal use, or for the internal or personal use of specific clients, beyond the fair use provisions granted by the U.S. Copyright Law is authorized by SPIE subject to payment of copying fees. The Transactional Reporting Service base fee for this volume is $\$ 18.00$ per article (or portion thereof), which should be paid directly to the Copyright Clearance Center (CCC), 222 Rosewood Drive, Danvers, MA 01923. Payment may also be made electronically through CCC Online at copyright.com. Other copying for republication, resale, advertising or promotion, or any form of systematic or multiple reproduction of any material in this book is prohibited except with permission in writing from the publisher. The CCC fee code is 0277$786 \mathrm{X} / 19 / \$ 18.00$.

Printed in the United States of America by Curran Associates, Inc., under license from SPIE.

Publication of record for individual papers is online in the SPIE Digital Library.

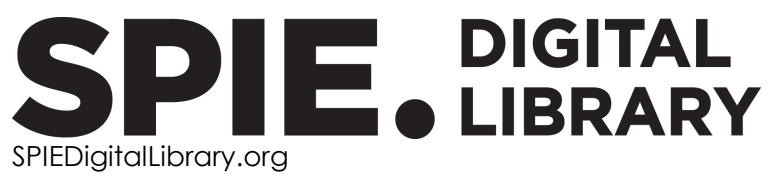

Paper Numbering: Proceedings of SPIE follow an e-First publication model. A unique citation identifier (CID) number is assigned to each article at the time of publication. Utilization of CIDs allows articles to be fully citable as soon as they are published online, and connects the same identifier to all online and print versions of the publication. SPIE uses a seven-digit CID article numbering system structured as follows:

- The first five digits correspond to the SPIE volume number.

- The last two digits indicate publication order within the volume using a Base 36 numbering system employing both numerals and letters. These two-number sets start with $00,01,02,03,04$, 05, 06, 07, 08, 09, 0A, OB ... 0Z, followed by 10-1Z, 20-2Z, etc. The CID Number appears on each page of the manuscript. 


\title{
Contents
}

\author{
vii $\quad$ Authors \\ ix Conference Committee
}

\section{STANDOFF SENSING OF CBRNE HAZARDS I}

1101002 CBRNE sensing at 20 years: a retrospection and prospects for the future (Keynote Paper) [11010-1]

1101003 Standoff hyperspectral imaging of CWAs and explosives using eyesafe QCL arrays [11010-2]

1101004 Infrared backscatter imaging spectroscopy for standoff detection of trace explosives [11010-3]

1101005 Real-time, reconfigurable, handheld molecular chemical imaging sensing for standoff detection of threats [1 1010-4]

1101006 Experimental considerations for the proximate standoff detection of highly scattering hazardous materials using infrared techniques [11010-5]

1101007 Dissemination monitoring by LWIR hyperspectral imaging [11010-6]

STANDOFF SENSING OF CBRNE HAZARDS II

1101008 Wide-area mapping and identification system for detection of chemical contamination on surfaces [11010-7]

1101009 Recent development of a UV Raman microscope explosive detection system for near trace detection [11010-9]

PHOTONIC INTEGRATED CIRCUIT AND PLASMONIC SENSORS

11010 OB Monolithically integrated silicon photonic chip sensor for near-infrared trace-gas spectroscopy [11010-11]

11010 OD Photo-induced enhanced Raman spectroscopy (PIERS): sensing atomic defects, explosives and biomolecules [11010-13]

11010 OF Approaching single molecule sensing: predictive sweat sensor design for ultra-low limits of detection [11010-15] 


\section{EXPLOSIVE SENSING}

$110100 \mathrm{G}$ Predicting the persistence of explosives materials [11010-16]

$11010 \mathrm{OH}$ Explosive detection and identification using a wide-area, hyperspectral Raman imaging sensor [11010-17]

1101001 Characteristics of trace explosives particles in fingerprints for optical detection [11010-18]

ADVANCES IN CBE SIGNATURE MODELLING AND SENSOR ALGORITHMS I

11010 OK Synthetic models for infrared reflectance signatures of micro-particle traces on surfaces [11010-20]

\section{ADVANCES IN CBE SIGNATURE MODELLING AND SENSOR ALGORITHMS II}

$110100 \mathrm{~L}$ A new, hand-held, 1- to 5-m standoff analyzer for real-time detection of trace chemical, biological, and explosive substances on surfaces [11010-8]

$110100 \mathrm{M}$ Obtaining the complex optical constants $\boldsymbol{n}$ and $\boldsymbol{k}$ via quantitative absorption measurements in $\mathrm{KBr}$ pellets [11010-21]

1101000 Accurate optical constants for liquids in the near-infrared: improved methods for obtaining the $n$ and $k$ constants from 1 to $4 \mu \mathrm{m}$ [11010-23]

\section{RADIOLOGICAL AND NUCLEAR SENSING}

11010 OR Hyperspectral vegetation identification at a legacy underground nuclear explosion test site [11010-26]

11010 OS Fixed-angle reflectance from uranium compounds and U-bearing minerals: experimental challenges to determine the optical constants $n$ and $k$ [11010-27]

REMOTE AND DISTRIBUTED SENSING OF CBRNE HAZARDS

11010 OT Development of technology for low-power gas sensing: IARPA's MAEGLIN program [11010-28]

11010 OU Remote vapor detection and classification using hyperspectral images [1 1010-29]

$110100 \mathrm{~V}$ From the warehouse to the field: new applications of existing chemical warfare agent detectors without hardware modification [1 1010-30] 
$110100 X \quad$ A new modality for the detection of chemical vapors using middle infrared femtosecond pulse propagation effects [11010-32]

$110100 Z$ Detecting atmospheric DHO with a spectroscopic THz sensor [11010-35]

\section{ADVANCES IN CHEMICAL HAZARDS SENSING}

$1101012 \mu$ ChemLab: twenty years of developing CBRNE detection systems with low false alarm rates [11010-38]

1101013 Passive LWIR hyperspectral imaging of surfaces contaminated by CWA droplets [11010-39]

1101014 Quantum cascade laser-based trace detection of gases in the deep-infrared region using phase fluctuation optical heterodyne spectroscopy [11010-40]

1101015 Fiber-lasers for coherent Raman detection of toxic chemical hazards [11010-41]

POSTER SESSION

1101017 Examination of stochastic and ordered methods to select optical filters for discrimination between chemical vibrational absorption bands [1 1010-43]

1101018 Infrared reflectance characterization of ammonium nitrate residue on roughened aluminum for potential bioinspired stand-off sensor [11010-44]

1101019 Angle-polished fibers as a robust coupling tool for photonics [1 1010-45]

$110101 \mathrm{~A} \quad$ Packaging of optofluidic microbubble resonator sensors [1 1010-46]

$110101 \mathrm{C} \quad$ Low SWaP chemical sensing via a fabric-based spectrometer [1 1010-48]

11010 1D Detecting radiation in a standoff geometry with mid-IR laser breakdown [1 1010-49]

$110101 \mathrm{E} \quad$ Remote detection of radioactive material using optically induced air breakdown ionization [11010-50] 
Proc. of SPIE Vol. 11010 1101001-6

\section{Downloaded From: https://www.spiedigitallibrary.org/conference-proceedings-of-spie on 25 Apr 2023
Terms of Use: https://www.spiedigitallibrary.org/terms-of-use}




\section{Authors}

Numbers in the index correspond to the last two digits of the seven-digit citation identifier (CID) article numbering system used in Proceedings of SPIE. The first five digits reflect the volume number. Base 36 numbering is employed for the last two digits and indicates the order of articles within the volume. Numbers start with 00, 01, 02, 03, 04, 05, 06, 07, 08, 09, 0A, OB...0Z, followed by 10-12, 20-2Z, etc.

Achuythan, Komandoor E., 12

Aggarwal, Ishwar D., 06, 17, 18

Alexander, Dennis R., OX

Anderson, Dylan Z., OR

Anderson, John M., 12

Andrews, Benjamin, 01

Ayhan, Bulent, OU

Banach, Catherine A., 0M, 00

Barwicz, Tymon, OB

Ben-Jaber, S., OD

Bhartia, R., OL

Bickford, Justin, OF

Blackman, C.S., OD

Blanchard, Romain, 03

Breshike, Christopher J., 04, OK

Brick, T., OD

Burton, S. D., OM

Cannon, Bret D., OS

Cantley, Lauren, 1C

Cho, Pak, OF

Clewes, Rhea J., 03

Collins, Adam D., OR

Colpoys, Caroline, 01

Coluccelli, N., 15

Conghuyentonnu, Thuyan, 09

Conner, Siobhan M., 03

Coppock, Matthew, OF

Corbey, Jordan F., OS

Cortés, E., OD

Craven, Julia M., OR

Dale, Elijah, $0 Z$

Demers, Joseph R., $0 Z$

DeVetter, Brent M., OS

DeWitt, Kristin, OT

Dominguez, Dawn, OK

Dong, Royce, 19, 1A

Dryer, Lars, 0 I

Duch, Elizabeth A., OB

Emge, Darren, 08

Emmons, Erik, 08

Ewing, Kenneth J., 06, 17, 18

Farrell, Mikella E., 06, OF

Fischer, Thomas, 01

Fix, Cory S., 12

Fountain, Augustus W., III, 02

Francis, Ryan M., OM, 00

Furstenberg, Robert, 04, 0G, 0I, OK, OM

Gambetta, A., 15

Glass, D., OD
Glodde, Martin, OB

Goldberg, Abby, OI

Gomer, Heather, $\mathrm{OH}$

Gomer, Nathaniel R., $\mathrm{OH}$

Graf, Darin, 12

Green, William M. J., OB

Guicheteau, Jason, 08

Harden, C. Steve, OV

Hardy, Hunter, 09

Harris, Jonathan, OF

Hauck, Brian C., OV

Holthoff, Ellen L., 06, OF

Howle, Chris R., 03, OD, 15

Huffman, Tyler J., OK

Hug, W. F., OL

Hung, Kevin, 08

Hutchens, Thomas C., 06, 17, 18

Ince, Brian S., OV

Isaacs, Joshua, 1D, 1E

Jensen, James O., OU

Johnson, Timothy J., OM, 0O, OS

Kelly-Gorham, Molly Rose K., OS

Kendziora, Christopher A., 04, OI, OK, OM

Kingsborough, Richard P., 1C

Krishnamurthy, Kalyani, 03

Kullander, F., 13

Kusterbeck, Andrew, 0G, OI, OK

Kwan, Chiman, $\mathrm{OU}$

Lambrakos, Samuel G., OK

Lamsal, Nirmal, $\mathrm{OH}$

Landström, L., 07, 13

Laporta, P., 15

Lee, Linda, 03

Liao, Jie, 19, 1A

Long, Stephen, OV

Loring, John S., 00

Maier, S. A., OD

Major, Kevin J., 06, 17, 18

Manginell, Ronald P., 12

Marchack, Nathan, OB

Martin, Yves, OB

McEwan, K., 15

McGill, R. Andrew, 04, 0G, Ol, OK

McHugh, Vincent M., OV

McIntrye, Henry, OV

McSweeney, Robert, OV

Milchberg, H. M., ID

Miller, Elizabeth D., OR

Molner, Tim, 09 
Moorman, Matthew W., 12

Myers, Edward B., 12

Myers, Tanya L., OM, 0O, OS

Nelson, Matthew P., 05, OH

Nguyen, Q., OL

Nguyen, Viet, 04, 0G, 01

Oeck, Ashley M., OM, 00

Orcutt, Jason S., OB

Osgood, Richard M., III, IC

Papantonakis, Michael, OG, OI

Parkin, I. P., OD

Pein, Brandt, 03

Pellegrino, Paul M., 06

Peveler, W. J., OD

Pfeifer, Kent, 12

Plaut, Maxwell, 1C

Polak, Adam, 14

Porter, Daniel, 12

Potter, Matthew G., 18

Poutous, Menelaos K., 17, 18

Qavi, Abraham J., 19, 1 A

Quesada-Cabrera, R., OD

Ratcliff, Erin L., OF

Redman, Brian J., OR

Reid, M., OL

Reid, R. D., OL

Resch, C. Tom, OS

Richards, John, 12

Riley, Patrick C., OV

Røen, B. T., 13

Roese, Erik, 08

Rothschild, Mordechai, $1 \mathrm{C}$

Roukes, Michael, 12

Sanghera, Jasbinder S., 06, 17, 18

Schares, Laurent, OB

Scharko, Nicole K., OS

Schultz-Fellenz, Emily S., OR

Schwartz, R. M., ID

Shabaev, Andrew, OK

Simonson, Robert J., 12

Sprangle, Phillip, 1D, 1E

Staton, Alan, 12

Stolyarov, Alexander, 1C

Stothard, David J. M., 14

Stroud, Lara, Ol

Su, Yin-Fong, 00

Sun, Haiyin, $\mathrm{OH}$

Swanson, Erika M., OR

Taylor, James, OV

Tazik, Shawna K., 05

Thomas, Jack W., 14

Thomas, William Conner, OX

Tjärnhage, T., 07

Tonkyn, Russell G., 00

Treado, Patrick J., 05

Trudeau, Robert, 1C

Tysk, Shane, 1C

Ulu, Gokhan, 03

Vakhshoori, Daryoosh, 03

van der Laan, John D., OR
Vander Rhodes, Greg, 03

Vicentini, E., 15

Vunck, Darius, 09

Washburn, Cody, 12

Wästerby, P., 07, 13

Waterbury, Rob, 09

Wheeler, David R., 12

Whiting, Joshua J., 12

Wilsenack, F., 07

Wilson, Christopher R., 18

Witinski, Mark F., 03

Wolf, T., 07

Woodbury, Daniel, 1D, 1E

Xiong, Chi, OB

Yang, Lan, 19, 1A

Yoon, Yohan, 04

Zehfus, Lilly, 0 I

Zhang, Eric J., OB

Zhu, Y., OD

Zuhlke, Craig A., OX 


\title{
Conference Committee
}

\author{
Symposium Chairs
}

Jay Kumler, JENOPTIK Optical Systems, LLC (United States)

Ruth Moser, Air Force Research Laboratory (United States)

Symposium Co-chair

John Pellegrino, Electro-Optical Systems Laboratory, Georgia Institute of Technology (United States)

Conference Chairs

Jason A. Guicheteau, U.S. Army Edgewood Chemical Biological Center (United States)

Chris R. Howle, Defence Science and Technology Laboratory (United Kingdom)

Conference Program Committee

Augustus Way Fountain III, U.S. Army Edgewood Chemical Biological Center (United States)

Russell G. Bartholomew, U.S. Army Edgewood Chemical Biological Center (United States)

Christopher C. Carter, Johns Hopkins University Applied Physics Laboratory (United States)

Darren K. Emge, U.S. Army Edgewood Chemical Biological Center (United States)

Anthony Esposito, Defense Threat Reduction Agency (United States)

Timothy J. Johnson, Pacific Northwest National Laboratory (United States)

Aaron LaPointe, U.S. Army Night Vision \& Electronic Sensors Directorate (United States)

Paul M. Pellegrino, U.S. Army Research Laboratory (United States)

Justin Bickford, U.S. Army Research Laboratory (United States)

Session Chairs

1 Standoff Sensing of CBRNE Hazards I

Jason A. Guicheteau, U.S. Army Edgewood Chemical Biological Center (United States)

2 Standoff Sensing of CBRNE Hazards II

Christopher R. Howle, Defence Science and Technology Laboratory (United Kingdom) 
3 Photonic Integrated Circuit and Plasmonic Sensors

Justin R. Bickford, U.S. Army Research Laboratory (United States)

4 Explosive Sensing

Aaron LaPointe, U.S. Army Night Vision \& Electronic Sensors Directorate (United States)

5 Advances in CBE Signature Modelling and Sensor Algorithms I

Darren K. Emge, U.S. Army Edgewood Chemical Biological Center (United States)

6 Advances in CBE Signature Modelling and Sensor Algorithms II

Darren K. Emge, U.S. Army Edgewood Chemical Biological Center (United States)

$7 \quad$ Radiological and Nuclear Sensing

Timothy J. Johnson, Pacific Northwest National Laboratory (United States)

8 Remote and Distributed Sensing of CBRNE Hazards

Jason Guicheteau, U.S. Army Edgewood Chemical Biological Center (United States)

Paul M. Pellegrino, U.S. Army Research Laboratory (United States)

9 Advances in Chemical Hazards Sensing

Christopher C. Carter, Johns Hopkins University Applied Physics

Laboratory, LLC (United States) 